CARLOS IGLESIAS-CRESPO

(University of Cambridge)

\title{
LA IMPORTANCIA DE LOS TRATADOS PSICOLÓGICOS ARISTOTÉLICOS PARA LOS ESTUDIOS RETÓRICOS
}

The Importance of Aristotle's Psychological Works for Rhetorical Studies

\section{A propósito de:}

Fred D. Miller Jr. (trad.), Aristotle: On the Soul and Other Psychological Works, Oxford: Oxford University Press, 2018, 276 pp. (ISBN 978-0-19-9588213).

\begin{abstract}
This note reviews the new translation of Aristotle's De Anima and Parva Naturalia by Fred D. Miller Jr. Against its backdrop, the importance of these psychological treatises for interdisciplinary, rhetorical research is also analyzed, with special emphasis on both Aristotelian criticism and the arts of memory from Antiquity to the early modern period.
\end{abstract}

KEY WorDS: Aristotle, De Anima, Parva Naturalia, rhetoric, memory.

RESUMEN: Esta nota reseña la nueva traducción del De Anima y los Parva Naturalia de Aristóteles firmada por Fred D. Miller Jr. A propósito de los textos, se analiza la importancia de estos tratados psicológicos para la investigación retórica interdisciplinar, tanto en la crítica aristotélica como en los estudios centrados en las artes de memoria desde la Antigüedad hasta la primera mitad de la Edad Moderna.

Palabras Clave: Aristóteles, De Anima, Parva Naturalia, retórica, memoria.

Fecha de Recepción: 20 de septiembre de 2020.

Fecha de Aceptación: 14 de octubre de 2020.

Ciertos sectores del mundo editorial europeo asisten desde hace unos años a una reedición o reactualización de los textos de la Antigüedad en los fondos de sus catálogos. ${ }^{1}$ En España este es el caso de la Nueva Biblioteca Clásica de Gredos, relanzada por RBA en 2019 con el objetivo de ofrecer, manteniendo las traducciones originales, introducciones convenientemente actualizadas y reenfocadas de los autores más importantes de la colección original. En el Reino Unido, este

${ }^{1} \mathrm{El}$ proyecto que ha generado estos resultados ha contado con el apoyo de una beca de la Fundación "la Caixa" (ID 100010434), cuyo código es LCF/BQ/EU20/11810016. 
movimiento está abanderado entre otros por la ya centenaria Oxford World's Classics. Tomemos por ejemplo el caso de Aristóteles. Desde la nueva traducción de la Física a cargo de Robin Waterfield en $2008,{ }^{2}$ contamos con nuevas ediciones de la Ética a Nicómano — una reedición de la traducción clásica de David Ross, revisada y comentada por Lesley Brown-, ${ }^{3}$ la Política —como en el caso anterior, una actualización de la traducción clásica de Sir Ernest Baker a cargo de R. F. Stalley-, ${ }^{4}$ sendas nuevas traducciones a cargo de Sir Anthony Kenny de la Ética a Eudemo - la primera traducción completa al inglés-y la Poética, ${ }^{5}$ así como nuevas traducciones de la Retórica, firmada por Robin Waterfield, ${ }^{6}$ y De Anima junto con los Parva Naturalia, a cuenta de Fred D. Miller Jr. ${ }^{7}$ Este último es el volumen que aquí nos ocupa.

El profesor Miller presenta una nueva traducción comentada que se caracteriza por los siguientes elementos formales. En primer lugar, las ediciones del texto griego utilizadas son las fijadas en su momento por W. D. Ross para Clarendon Press. ${ }^{8}$ Aunque estas son seguidas con fidelidad, Miller no duda en corregir su traducción y por consiguiente el texto base de Ross- en aquellos lugares en los que cree necesario armonizar la edición con lecturas concretas a partir de los manuscritos conservados. En segundo lugar, Miller opta por una traducción más literaria que literal al inglés, debido a que está pensada para un ámbito más amplio de lectores que no conozcan el griego en profundidad o que no tengan una formación previa en Clásicas. ${ }^{9}$ Sin embargo, ello no quiere decir que el traductor no conserve en esta versión elementos del estilo silogístico característico del estagirita. Al contrario, Miller toma grandes precauciones para asegurar que la traducción de estas complejas obras fluya de forma natural, pero con fidelidad al estilo argumentativo y al discurrir lógico que les imprimió Aristóteles.

En lo que respecta al contenido, este volumen está estructurado de un modo acorde con la práctica habitual de la colección Oxford World's Classics. A saber: cuenta con una introducción, una nota sobre el texto y la traducción, una bibliografía, una cronología, la traducción en sí misma, un comentario, un glosario y un índice final.

La introducción ocupa unas cincuenta páginas en las que el profesor Miller introduce toda una serie de consideraciones relevantes. ${ }^{10}$ La primera oración deja

\footnotetext{
2 Aristóteles (2008).

3 Aristóteles (2009a).

${ }^{4}$ Aristóteles (2009b).

${ }^{5}$ Aristóteles (2011) y (2013).

${ }^{6}$ Aristóteles (2018a).

${ }^{7}$ Aristóteles (2018b).

${ }^{8}$ Ross (1955) y (1956).

${ }^{9}$ Miller (2018: lx).

${ }^{10}$ Miller (2018: xi-lix).
} 
claros los derroteros por los que esta disertación va a discurrir: "Aristotle (...) may rightly be called the founder of psychology, because he was the first to undertake a systematic investigation in what is now known as the science of mind and behaviour". ${ }^{11}$ Esta supone toda una declaración de intenciones que se explicita en la exposición del tratado, en el que la tesis de Miller se apoya en dos pilares. Por una parte, un pilar científico, basado en la tesis de que los temas que toca Aristóteles son afines a la distribución de los libros de texto modernos sobre psicología, dada la atención que presta a conceptos clave como la sensación, la percepción, la imaginación, la memoria, la cognición, la motivación, el deseo y procesos fisiopsicológicos de diversa índole. ${ }^{12}$ Por otra, un pilar filosófico que contextualiza el texto aristotélico dentro de los marcos de la filosofía de la mente y de la fenomenología. ${ }^{13}$ En base a estos dos importantes pilares temáticos, Miller concluye que el estagirita "still offers valuable insights into the explanation of psychological processes and capacities". ${ }^{14}$ Este supone el punto de vista desde el que este volumen está concebido: presentar a Aristóteles dentro de una genealogía intelectual o historia de las ideas concreta, a saber, la historia de la ciencia y la historia de la filosofía. No obstante, la introducción no se limita al tratamiento de Aristóteles como un precursor dentro de una larga cadena que se prolonga en las siguientes centurias, sino que defiende que la sombra del De Anima y los tratados menores todavía es larga y debe ser tenida en cuenta por su influencia en diferentes ámbitos.

Es por ello por lo que Miller no se contenta con tratar las cuestiones habituales en las introducciones divulgativas escritas por académicos —a saber, la vida del filósofo, una breve descripción de su sistema filosófico y un análisis detallado de las secciones del tratado-, sino que lanza un guante a los lectores como razón de ser de esta nueva traducción. En las últimas páginas de la introducción se nos presenta toda una batería de cuestiones que surgen a propósito o en respuesta a la teoría aristotélica frente a la postura contraria defendida por Burnyeat. Estas cuestiones van desde la posibilidad de aplicar una perspectiva hilomórfica a la neurociencia moderna hasta lo apropiado de un acercamiento teleológico en el análisis del desarrollo fisiológico y psicológico, sin olvidar el planteamiento de otros tipos de cuestiones de corte cognitivo y conductual de especial interés hoy en día. Es por ello mismo por lo que

11 Miller (2018: xi).

12 Miller (2018: xi-xii). Véase Bloch (2007: 128-135) para una aproximación a la influencia indirecta de Aristóteles en las teorías modernas de memoria. Véase también Glannon (2019: capítulo 1) para una visión diacrónica de la evolución de las teorías sobre la memoria desde Aristóteles hasta nuestro tiempo: "Although concepts of memory from Aristotle and Augustine may seem relatively simple by today's standards, they contain elements that are precursors of how researchers now conceive of memory".

13 Miller (2018: xii). Véase por ejemplo el uso que Paul Ricoeur (2004: capítulo 1) hace de las observaciones aristotélicas como cimiento para su fenomenología de la memoria.

${ }^{14}$ Miller (2018: xii). 
Miller afirma que "The aim of the present volume is to provide readers with Aristotle's texts in translation together with scholarly resources need in order to start seeking the answers to such questions". ${ }^{15}$

No obstante, a pesar del enfoque que estamos resaltando, hemos de afirmar que Miller no peca en ningún momento de un acercamiento anacrónico al texto, que supedite a Aristóteles a nuestro tiempo, sino que en todo momento contextualiza al filósofo dentro de su propio sistema y momento histórico-uno de los aspectos más señeros de la introducción es la visión de conjunto que ofrece al poner en relación los tratados psicológicos con el resto del corpus aristotélico: Retórica, Poética, Ética a Nicómano, Metafísica, Física, Partes de los animales, Segundos analíticos, etc.-, y nos lo presenta como una ventana al pasado, pero desde la cual podemos evaluar posturas teóricas del presente.

Tras la introducción y la nota textual, el volumen ofrece una bibliografía selecta de textos editados y traducciones, manuscritos, comentarios clásicos y medievales, y una amplia bibliografía secundaria, así como recomendaciones para profundizar con los temas tratados, ${ }^{16}$ desde monográficos recientes como la traducción y trabajos de Bloch sobre el De Memoria, ${ }^{17}$ pasando por la colección de ensayos de Nussbaum y Rorty sobre De Anima, ${ }^{18}$ la traducción y comentario de Gallop de De Somno y De Insomniis, ${ }^{19}$ a las ediciones ya mencionadas de Ross que son la base del texto traducido. Por otra parte, cabe mencionar que otro de los grandes atractivos de esta traducción son sus apéndices, que ofrecen todo un catálogo de testimonios y fragmentos — entre otros, pasajes que dan cuenta del escrito perdido De Philosophia, así como una traducción del Himno a Hermias, atribuido a Aristóteles y que Miller presenta como un colofón adecuado a los tratados sobre el alma.

Los apartados finales del libro están dedicados al comentario del texto. Las notas son por lo general breves y escuetas, de gran concisión. Quizás el mayor acierto es el ejercicio de referencia cruzada que realiza, no solo entre las diversas secciones del De Anima-Miller anota cada vez que Aristóteles hace referencia a temas ya mencionados-, sino también entre el De Anima y los diversos Parva Naturalia, así como con otros textos del corpus que pudieran ser de utilidad para el investigador o el curioso. Estas se ven respaldadas por un breve aparato crítico con el que Miller justifica sus óbelos respecto a la edición de Ross, así como un útil glosario de términos transliterados y las elecciones concretas del traductor para unificar la

\footnotetext{
15 Miller (2018: lix).

16 Miller (2018: 1xi-lxix).

17 Bloch (2007).

18 Nussbaum y Rorty (1995).

19 Gallop (1990).
} 
traducción. Cada elección cuenta con una explicación al respecto con referencias cruzadas al aparato o al comentario.

Esta es, en definitiva, una buena traducción que destaca por su utilidad de corte escolar que no olvida un propósito investigador, si bien siempre manteniendo una encomiable postura divulgativa. Esto hace del texto de Miller una opción atractiva debido tanto a su rigor como a su accesibilidad, así como por el gran número de cabos y retos que lanza al lector a propósito de pasajes relacionados con los analizados que se encuentran esparcidos por el conjunto los tratados aristotélicos.

$\mathrm{Y}$ es con vistas a esta visión sistemática que cabe enfatizar la importancia que tienen el texto de De Anima y de los Parva Naturalia para un estudiante de la retórica aristotélica y de su influencia posterior. La crítica especializada de un tiempo a esta parte ha realizado lecturas cada vez más interdisciplinares y sistemáticas de la Retórica aristotélica. Ejemplo de ello son los trabajos de James J. Murphy, Sara Newman, Debra Hawhee, Krisanna Scheiter o Monica Westin. Aunque, naturalmente, cada investigador tiene su propio proyecto, los arriba mencionados coinciden en la importancia capital que tienen los tratados psicológicos aristotélicos para comprender sus postulados retóricos. Murphy explica cómo la retórica requiere de una "metaretórica", donde las nociones de percepción, phantasía, memoria, expuestas en De Anima y en los Parva Naturalia son claves para comprender los elementos involucrados en el acto discursivo. ${ }^{20}$ Scheiter, por su parte, realiza un ejercicio esclarecedor sobre la función de la phantasía en la percepción sensitiva de las imágenes mentales propias tanto del razonamiento psicológico como del discurso. ${ }^{21}$ Newman y Hawhee señalan la deuda ineludible que tiene la Retórica con la psicología aristotélica para poder explicar cognitivamente la enérgeia, la capacidad para $\pi \rho \grave{~}$

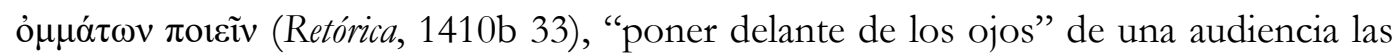
imágenes metafóricas de las que el orador echa mano en su discurso. ${ }^{22}$ Westin, por su parte, va un paso más allá, demostrando cómo la noción de enérgeia es consistente a lo largo y ancho del corpus aristotelicum, y como tal es necesaria una visión de conjunto que abarque textos tan dispares como la Metafísica, la Retórica o el De Anima para poder entender su uso con propiedad en cada caso particular. ${ }^{23}$

Asimismo, es necesario resaltar que el De Memoria et Reminiscentia en particular, uno de los siete Parva Naturalia, tiene una importancia fundamental para los investigadores volcados en el estudio de la memoria como parte de la retórica. Recordemos que este tratado psicológico presenta una distinción entre la memoria, que tiene como objeto el recuerdo, y la reminiscencia, que tiene como función la

\footnotetext{
20 Murphy (2002).

21 Scheiter (2012).

22 Newman (2002) y Hawhee (2011).

23 Westin (2017).
} 
recuperación del recuerdo. ${ }^{24}$ Aristóteles construye un sistema de memoria artificial que agiliza los procesos de reminiscencia, basado en cadenas ordenadas de razonamiento con base en procesos de asociación conceptual y lugares mentales. ${ }^{25}$ En su comentario, Miller identifica este sistema con las artes de memoria retóricas a las que Aristóteles parece referirse también en De Anima, donde el estagirita

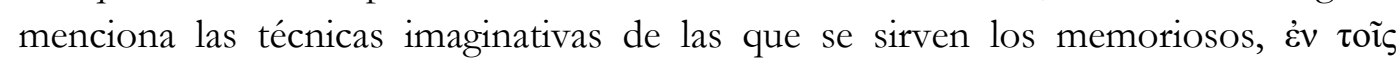
$\mu \nu \eta \mu о v к к о \tilde{\varsigma}$ (427b 17-21). ${ }^{26}$ Sin la memoria artificial aristotélica no se entiende buena parte de la tradición de las artes de memoria desde la Antigüedad hasta el Barroco europeo, como ha sido demostrado en los numerosos trabajos dentro de este campo a partir del estudio ya clásico de Yates. ${ }^{27}$

En el contexto de la Roma republicana e imperial, las secciones dedicadas a la memoria y sus artes en la anónima Rhetorica ad Herennium (3.28-3.40), el De Oratore (2.51-2.60) de Cicerón y las Institutio Oratoria (11.2.1-11.2.51) de Quintiliano contraen una clara deuda con los principios mnemotécnicos aristotélicos. ${ }^{28}$ En la Edad Media pre-escolástica, la concepción aristotélica de la memoria sobrevive en los comentarios que Avicena y Averroes hacen tanto del De Anima como de los Parva Naturalia. ${ }^{29}$ Sin embargo, esta gozará de una arrolladora vigencia filosófica y retórica a partir de la explosión escolástica del siglo XIII, cuando San Alberto Magno y Santo Tomás de Aquino ofician el matrimonio entre Aristóteles y la Rhetorica ad Herennium, ${ }^{30}$ presente en el arte de memoria de Jorge de Trebisonda. ${ }^{31}$ En el Renacimiento y en el Barroco, la memoria aristotélica, para siempre unida a la escritura per locos et imagines hereniana, deja una fuerte impronta en contextos muy variados. ${ }^{32}$ Por ejemplo: en la España de distinción

24 Aristóteles (451a 14-25). Véase Bloch (2007: 75-77) sobre las implicaciones de esta

25 Aristóteles (451b 13-22 y 452a 13-30).

${ }^{26}$ Polansky (2007: 411) y Miller (2018: 225). Aunque innegablemente Aristóteles construye un modelo de memoria artificial que es uno de los precursores de las artes de memoria, es bastante probable que no tuviera una concepción retórica de las mismas como artes de memoria propiamente dichas, dado que la memoria no estaba codificada como parte de la retórica en época clásica. Como Calboli (2020: 493) observa, no es hasta la Rhetorica ad Herennium que encontramos en la tradición grecolatina el primer ejemplo que conservamos de un arte de memoria dentro de la memoria como officium oratoris, lo que nos lleva necesariamente a un campo de investigación post-aristotélico.

27 Yates (1999).

28 Yates (1999: capítulo 1), Zarzo (2016: 92-99). Se ha de notar que Quintiliano adopta una postura un tanto escéptica, incluso contraria, respecto a la utilidad del sistema per locos et imagines, lo que contrasta con la clara aprobación del sistema de la que hace gala la Rhetorica ad Herennium (Merino Jerez 2007: 46).

${ }^{29}$ Coleman (1992: 401-415), Bloch (2007: 144-145).

30 Carruthers (2008: 155).

31 Merino Jerez (2007: 67-68).

32 Merino Jerez (2007: 49-52). Es cierto, no obstante, que la corriente aristotélica de la memoria tuvo que competir con influyentes tendencias platónicas, neoplatónicas, herméticas y cabalísticas, que cuentan con figuras tan señeras para la historia de la mnemotecnia como Ramón Llull, Giordano Bruno o Giulio Camillo. Véase Merino Jerez (2007: 44-45) para las diferencias entre el 
los siglos XVI y XVII, esta aparece en obras misceláneas, como la Silva de varia lección de Pedro Mexía; ${ }^{33}$ en las artes de predicación y tratados de meditación de los jesuitas, como los Ejercicios espirituales de San Ignacio de Loyola; ${ }^{34}$ en el Comulgatorio de Baltasar Gracián; ${ }^{35}$ en las anotaciones de Herrera al Soneto VIII de Garcilaso; ${ }^{36}$ en la obra médica de Juan Gutiérrez de Godoy; ${ }^{37}$ en los programas de estudios de universidades como la de Alcalá de Henares, donde el De Anima y los Parva Naturalia, junto con sus comentarios escolásticos, estaban en la base de la enseñanza en arte y filosofía; ${ }^{38}$ y en artes de memoria propiamente dichas, como es el caso del Fénix de Minerva o arte de memoria de Juan Velázquez de Azevedo. ${ }^{39}$

Así pues, resulta evidente que los estudios retóricos —estén estos especializados en la obra de Aristóteles, rastreen su impacto y recepción a lo largo de los siglos, o analicen la construcción y principios retóricos de un abanico tan variado de obras como las arriba mencionadas- deben estar íntimamente familiarizados con los contenidos de los tratados psicológicos del estagirita. Por tanto, en este contexto de investigación, una nueva traducción del De Anima y los Parva Naturalia tan clara y esclarecedora como la que nos presenta Miller no puede ser más bienvenida.

\section{CARLOS IGLESIAS-CRESPO ci244@cam.ac.uk Queens' College University of Cambridge}

aristotelismo y el platonismo mnemotécnico; Bolzoni (2001: 237-259) para el desarrollo de las tendencias neoplatónicas en el siglo XVI; y Cacho Casal (2009: 1168-1171) sobre algunos usos renacentistas de la memoria para la reconstrucción topológica e imaginaria de Roma.

33 Mexía (1990: 48-60). Véase también Egido (1986: 25-26) y Merino Jerez (2007: 49).

34 Rodríguez de la Flor (1996: 113-122). Véase también Rodríguez de la Flor (1996: capítulo 2) para un tratamiento extenso del impacto que tuvieron las concepciones clásicas de la memoria retórica en la imaginería religiosa de la Contrarreforma.

35 Rodríguez de la Flor (1996: 155). Véase también Egido (1986) para un estudio de la importancia de la memoria en el Criticón.

${ }^{36}$ Herrera (2001: 338). Véase Ramos (2007) para una aproximación al papel de la memoria en el quehacer estético del poeta sevillano.

${ }^{37}$ Rodríguez de la Flor (2002: xlvii).

38 Jauralde Pou (1998: 110), por ejemplo, ilustra cómo un joven Quevedo tuvo que leer un buen número de obras de Aristóteles, entre ellas los tratados psicológicos, a su paso por esta universidad entre 1596 y 1599. Véase Alvar Ezquerra (1996) para una visión panorámica de la Universidad de Alcalá a principios del siglo XVI. Véase también Cacho Casal (2009) para el uso que Quevedo hace de las artes de memoria en su silva "Roma antigua y moderna".

${ }^{39}$ Merino Jerez (2007: 50). Véase el estudio introductorio de Rodríguez de la Flor (2002) para un análisis de la ecléctica encyclopaedia mnemonica que fundamenta la obra de Velázquez de Azevedo. 


\section{BIBLIOGRAFÍA}

Alvar Ezquerra, A. (1996), La Universidad de Alcalá de Henares a principios del siglo XVI, Madrid: Servicio de Publicaciones de la Universidad de Alcalá.

Aristóteles (2008), Physics, trad. Robin Waterfield, introducción y notas de David Bostock. Oxford: Oxford University Press.

Aristóteles (2009a), The Nicomachean Ethics, nueva edición, trad. D. Ross, ed. Lesley Brown. Oxford: Oxford University Press.

Aristóteles (2009b), Politics, trad. Sir Ernest Baker, revisión, introducción y notas de R. F. Stalley. Oxford: Oxford University Press.

Aristóteles (2011), The Eudemian Ethics, trad. Anthony Kenny, Oxford: Oxford University Press.

Aristóteles (2013), Poetics, trad. Anthony Kenny, Oxford: Oxford University Press.

Aristóteles (2018a), The Art of Rhetoric, trad. Robin Waterfield, Oxford: Oxford University Press.

Aristóteles (2018b), On the Soul and Other Psychological Works, trad. Fred. D. Miller Jr., Oxford: Oxford University Press.

Bloch, D. (2007), Aristotle on Memory and Recollection, Leiden: Brill.

Bolzoni, L. (2001), The Gallery of Memory. Literary and Iconographic Models in the Age of the Printing Press, trad. J. Parzen, Toronto: University of Toronto Press.

Cacho Casal, Rodrigo (2009), “The Memory of Ruins: Quevedo's Silva to "Roma antigua y moderna'”, Renaissance Quarterly 62(4): 1167-1203.

Calboli, G. (2020), Cornifici seu Incerti Auctoris Rhetorica ad C. Herennium: Prolegomena, ed., trad. y comentario, Berlin; Boston: De Gruyter.

Carruthers, M. (2008), The Book of Memory: A Study of Memory in Medieval Culture, $2^{\mathrm{a}}$ edición, Cambridge: Cambridge University Press.

Coleman, J. (1992), Ancient and Medieval Memories: Studies in the Reconstruction of the Past, Cambridge: Cambridge University Press.

Egido, A. (1986), "El arte de la memoria y El Criticón”, en Gracián y su época: actas de la I Reunión de Filólogos Aragoneses: ponencias y comunicaciones, Zaragoza: Institución Fernando el Católico, pp. 25-66.

Gallop, D. (1990), Aristotle on Sleep and Dreams, texto, traducción y notas, Peterborough, Ontario: Broadview Press.

Glannon, W. (2019), The Neuroethics of Memory: from Total Recall to Oblivion, Cambridge: 


\section{TALIA DIXIT 15 (2020), 123-132}

Cambridge University Press.

Hawhee, D. (2011), “Looking Into Aristotle's Eyes: Toward a Theory of Rhetorical Vision", Advances in the History of Rhetoric 14(2): 139-165.

Herrera, F. de (2001), Anotaciones a la poesía de Garcilaso, ed. Inoria Pepe y José María Reyes, Madrid: Cátedra.

Jauralde Pou, P. (1998), Francisco de Quevedo (1580-1645), Madrid: Castalia.

Merino Jerez, L. (2007), Retórica y artes de memoria en el Humanismo renacentista (Jorge de Trebisonda, Pedro de Ravena y Francisco Sánchez de las Brozas), Cáceres: Universidad de Extremadura.

Mexía, P. (1990), Silva de varia lección, II, ed. A. Castro Díaz, Madrid: Cátedra.

Miller, Jr., F. D. (2018), “Introduction”, en Aristóteles (2018b), On the Soul and Other Psychological Works, trad. F. D. Miller Jr., Oxford: Oxford University Press, pp. xi-lix.

Murphy, J. J. (2002), “The Metarhetoric of Aristotle, with Some Examples from His "On Memory and Recollection", Rhetoric Review 21(3): 213-228.

Nussbaum, M. y Rorty, A. O. (eds.) (1995), Essays on Aristotle's De Anima, Oxford: Oxford University Press.

Newman, S. (2002), “Aristotle's Notion of “Bringing-before-the-Eyes": Its Contributions to Aristotelian and Contemporary Conceptualizations of Metaphor, Style, and Audience", Rhetorica: A Journal of the History of Rhetoric 20 (1): 1-23.

Polansky, R. (2007), Aristotle's De Anima, Cambridge: Cambridge University Press.

Ramos, G. (2007), "Estética de la memoria en torno a Fernando de Herrera", en L. Fernando Lara, R. Yunuen Ortega y M. Lilia Tenorio (eds.), De amicitia et doctrina. Homenaje a Martha Elena Venier, Ciudad de México: Colegio de México, pp. 299-311.

Ricoeur, P. (2004), Memory, History, Forgetting, trad. K. Blamey y D. Pellauer, Chicago; London: The University of Chicago Press.

Rodríguez de la Flor, F. (1996), Teatro de la memoria. Siete ensayos sobre mnemotecnia española de los siglos XVII y XVIII, $2^{a}$ edición, Salamanca: Junta de Castilla y León.

Rodríguez de la Flor, F. (2002), "Estudio introductorio", en F. Velázquez de Azevedo, Fénix de Minerva o arte de memoria, Valencia: Tératos.

Ross, W. D. (1955), Aristotle. Parva Naturalia, Oxford: Clarendon Press.

Ross, W. D. (1956), Aristotelis De Anima, Oxford: Clarendon Press.

Scheiter, K. M. (2012), "Images, Appearances, and Phantasia in Aristotle", Phronesis 57(3): 251-278. 
Velázquez de Azevedo, F., Fénix de Minerva o arte de memoria, Valencia: Tératos.

Yates, F. (1999), Selected Works, Volume III: The Art of Memory, primera edición 1966, London; New York: Routledge.

Zarzo, E. (2016), Memoria retórica y experiencia estética. Retórica, Estética y Educación, Madrid: Dyckinson. 